\title{
Factors Determining the Choice of Vegetarian vs. Meat-Eating Diets
}

\author{
Alexie C. Maxwell ${ }^{1}$, Arianna M. Smith ${ }^{1}$, Emily R. Thomas ${ }^{1}$, Samuel P. Abraham ${ }^{1} \&$ Deborah R. Gillum ${ }^{1}$ \\ ${ }^{1}$ Bethel University School of Nursing, Mishawaka, Indiana, USA \\ Correspondence: Samuel P. Abraham, Bethel University School of Nursing, 1001 Bethel Circle, Mishawaka, \\ Indiana, 46545, USA.
}

Received: May 27, 2019

Accepted: June 12, 2019

Online Published: June 21, 2019

doi:10.20849/ijsn.v4i3.598

URL: https://doi.org/10.20849/ijsn.v4i3.598

\begin{abstract}
Background: There are various opinions of what eating healthy looks like, and it has become a frequent topic of discussion among young adults. Purpose: The purpose of this study was to explore the factors determining the choice of vegetarian vs. meat-eating diet. Method: This study was a qualitative phenomenological study with a descriptive design that was conducted at a college in northern Indiana area using one-on-one interviews. The sample size was 16 individuals, 8 who use the vegetarian diet, and 8 who are meat-eaters. Pender's health promotion model was used to guide this study. Results: There were 9 themes that emerged, of which 5 were vegan/vegetarian and 4 were meat-eaters. The vegetarian and vegan themes were limitation of the diet (1), health effects (2), confusion and stigma about diet (3), reasons for choosing this diet (4), and more conscious of what they eat (5). The meat-eating themes were choices (1), health effects (2), mocking and ridiculing (3), and workout (4). Conclusion: Overall, meat-eaters had more choices in their diet and vegetarians and vegans struggled with limitations. They discussed mocking or stigma about their diets as well as health effects. The need for further studies on this topic is evident from the literature review.
\end{abstract}

Keywords: vegan, vegetarian, health benefits, plant-based diet, effects of vegan/vegetarian diet

\section{Introduction}

There are various opinions of what eating healthy looks like, and it has become a frequent topic of discussion among young adults. Over the years, the vegan and vegetarian diets are a trending healthy lifestyle choice (Cramer et al., 2017). As these discussions and transitions of diet occur, it is important to be informed of the reasons why people make these diet choices. The purpose of this qualitative study was to explore the factors determining the choice of vegetarian vs. meat-eating diet.

\subsection{Background}

Over the years, the vegetarian dietary choices have grown increasingly popular. Cramer et al. (2017) "found that the prevalence of health vegetarianism (including health veganism) in this nationally representative sample was $1.9 \%$ in 2012, which was an $18.8 \%$ increase from 2002" (p. 564). While there is a stark increase in individuals who follow these diets, there is still a lack of research factors that influence these choices. This shift requires attention as to why the increase in this dietary lifestyle. Most research on vegetarian diets were describing the health benefits or effects of these diets. Many studies indicated there was limited research done on plant-based diets and that more need to be conducted. For instance, Brown (2018) conducted a literature review on the effects of the plant-based diets on dancers while Godos, Bella, Sciacca, Galvano, and Grosso's (2017) studied cancer risk and plant-based diets and both concluded a need for more research in this area.

There was specifically a lack of research on factors that influence the choice of a vegetarian diet. The study that examined these factors only explored the health reasons to follow these diets. Cramer et al. (2017) stated, "Missing from this survey was the assessment of whether individuals minimally consume meat and/or follow a full plant-based diet for reasons other than health" (p. 566). Many studies found nutrition has an impact on health; therefore, the large change in diet affects the nursing care given to patients. The purpose of this qualitative study was to explore the factors determining the choice of vegetarian vs. meat-eating diets.

\subsection{Research Question/s}

RQ1. What are the factors determining the choice of a vegetarian diet?

RQ2. What are the factors determining the choice of a meat-eating diet? 


\subsubsection{Interview Questions}

- $\quad$ For vegetarians: Tell me your personal experiences with your vegetarian diet. Please describe in detail.

- For meat-eaters: Tell me your personal experiences with your typical diet. Please describe in detail.

1.2.2 Prompts

- What influenced you to choose this diet?

- Who influences you to follow this diet (friends, family, etc.)?

- How does this affect your physical health?

- How does this affect your emotional health?

- How do you manage your diet when you are among friends who do not follow this diet?

- What experience do you have about people mocking or ridiculing your dietary preferences?

- What challenges do you find in following your preferred diet?

\section{Review of the Literature}

A thematic review of relevant literature is discussed below. Search procedures included the use of the Cumulative Index of Nursing and Applied Health Literature (CINAHL) and EBSCOhost databases. Keywords used to find these articles were vegan, vegetarian, health benefits, plant-based diet, and effects of vegan/vegetarian diets. Articles written from 2015 to 2018 were reviewed along with relevant studies found in the reference lists.

\subsection{Transitions to Vegetarian Diets for Health Reasons}

There are a few reasons for people transitioning to the vegetarian or vegan diet; those including moral, ethical, spiritual, or religious beliefs, as well as those concerning animal cruelty (Cramer et al., 2017, p. 1). Along with these reasons, a major factor for transitioning into this diet is the perception of its health benefits. There have been a number of studies that have indicated that the vegetarian or vegan diet might be "associated with a reduction of weight, a lower incidence of the metabolic syndrome or diabetes, improvements in blood pressure and dyslipidemia and a lower incidence and/or mortality related to ischemic heart disease" (Cramer et al., 2017, p. 1). In addition to this, Dinu, Abbate, Gensini, Casini, and Sofi (2017) found that vegetarian and vegan diets show "significantly lower levels of the most relevant risk factor for chronic disease, such as body mass index (BMI), lipid variables and fasting glucose," as compared to a meat-eating diet (p. 5).

The American Dietetic Association (ADA) has promoted the switch to vegan or vegetarian diets as they claim them to be nutritionally sufficient, healthy, and contribute to health and the treatment and prevention of specific diseases (Piccoli et al., 2015). The ADA also verified that remaining on a vegan or vegetarian diet while pregnant is a healthy decision and will not be nutritionally depleting for pregnant mothers or the unborn fetus. Piccoli et al. reported that the prevalence of 'toxemia,' or pregnancy-induced hypertension, decreased to $4 \%$ in vegetarians compared to omnivores at $12 \%$.

Generally, it is difficult to fully account for the perceived health benefits of the vegetarian diet alone as people who tend to follow this diet also engage in other factors, such as exercise. It is apparent though that vegetarians have a better metabolic profile that should theoretically help prevent chronic degenerative diseases (Chiu et al., 2015, p. 1). The vegetarian or vegan diets often consist of eating less saturated fats and dietary cholesterol, which overall leads to a reduction of low-density lipoproteins (LDLs) (Trepanowski \& Varady, 2015, p. 7). The significance of these results is that vegetarian and vegan diets help improve blood lipids and help prevent chronic degenerative diseases.

\subsection{Health Benefits}

Many studies examined the health benefits from vegetarian diets. One study found that "with a cautious management of carbohydrate and fructose intake, plant-based diets can benefit all aspects of the metabolic profile" (Chiu et al., 2015, p. 1319). Similarly, Trepanowski and Varady (2015) found that when following a vegan diet, participants had lower cholesterol levels, decreased body weight, lower blood pressure, and decreased glucose and insulin levels as compared with individuals following a meat-eating diet. Dinu et al. (2017) stated that compared with meat-eaters, vegetarians and vegans tend to have lower BMI, glucose levels, and lipid levels.

Another benefit of the vegan or vegetarian diet is related to a decreased risk for cancer. Cramer et al. (2017) found that these diets improved heart health and decreased risk of cancer when compared to the general meat-eating diet. Godos et al. (2017) found a similar result of reduced risk of cancer when people followed diets that contained mainly fruits and vegetables. These studies described benefits in metabolic panels, as well as a reduced risk of 
cancer for those following a vegan or vegetarian diet when compared with those following an omnivorous diet.

\subsection{Effects on Physical Performance}

Two studies examined the effect of vegetarian diets on physical performance. Craddock, Probst, and Peoples (2016) found that there was no overall difference "in muscular power, muscular strength, anaerobic or aerobic performance between omnivorous or vegetarian athletes" (p. 219). Brown (2018) stated these diets could help to provide energy needed for athletes; however, those who follow a vegan diet need to ensure they are consuming the micronutrients that their bodies require.

\subsection{Summary of the Literature Review}

The review of literature contained several themes: transitions to vegetarian diets for health reasons, health benefits, and effects on physical performance. The research indicates that many people switch to a vegan or vegetarian diet for health reasons (Cramer et al., 2015). Health benefits included reduced risk of certain cancers (Cramer et al., 2017) and a better metabolic panel (Chiu et al., 2015). There were no differences in physical performance between omnivorous and vegetarian athletes (Craddock, Probst, \& Peoples, 2016). This review of literature revealed a gap in the research concerning factors that influence people's choices regarding vegetarian vs. meat diets.

\subsection{Theoretical Framework}

Pender's health promotion model (HPM) was be used to guide this study. The HPM identifies the factors that influence health behavior, specifically focusing on eight beliefs that can be assessed by the nurse. These eight beliefs are considered critical points for nursing interventions. Using the model and working collaboratively with the patients, the nurse can assist the client in changing behaviors to achieve a healthy diet (Pender, Murdough, \& Parsons, 2006).

\subsubsection{Pender's Health Promotion Model}

The HPM is based on the foundation of behaviors people adopt for their health (Pender et al., 2006). It fits well as a framework for this study because this research was an assessment of health benefits considered by people who follow a vegetarian diet compared to people who choose to include meat in their diet. This model has eight core beliefs that help the nurse to access the behaviors and to fully determine effectiveness and interventions. Through analysis of vegetarian beliefs, the nurse can discover the perceived benefits of this diet to promote a healthy diet. Conversely, the nurse can determine the barriers, such as what challenge this lifestyle may have. Through the HPM, the nurse can discover other contributing reasons a person may follow this diet and how they may stay firm in this diet decision. Overall, this model fits this study well and can help the nurse understand why certain diet choices, such as following a vegetarian or meat-eating diet, might be chosen.

\subsubsection{Definition of Terms}

\subsubsection{Operational Definitions}

The vegan diet is defined as a diet practice based on the avoidance of anything deriving from animal sources; some examples would be avoiding meat, milk products, and leather. The vegetarian diet is a diet choice on the avoidance of the consumption of meat products in their diets. Meat-eating diet is defined as a diet that has no restrictions on the consumption of meat; however, some may choose to eat certain meats and avoid others for religious or personal reasons. College students are individuals who are currently attending or recently graduated college; age ranges varying from 18-27 years old.

\subsubsection{Conceptual Definitions}

Pender et al. (2006) defined perceived benefits of action as the anticipated positive outcomes that will occur from health behaviors. Perceived barriers to action are defined as anticipated, imagined or real blocks, and include personal costs of understanding a given behavior. Perceived self-efficacy is a person's personal judgment of capability to plan and perform a health-promoting behavior. Activity-related affect is defined as a subjective positive or negative feeling that occurs before, during, and following behavior. Interpersonal influences are the beliefs concerning behaviors, beliefs, or attitudes of others. These influences include norms (expectations of significant others), social support (encouragement), and modeling (vicarious learning through observing); the primary sources of influences are family, peers, and healthcare providers. Situational influences are defined as personal perceptions and ideas of any given situation or context that can facilitate behaviors (Pender et al., 2006).

\section{Methodology}

\subsection{Method and Design Appropriateness}

This study was a qualitative, phenomenological study with a descriptive design. The interviews were regulated 
using Colaizzi's strategy. The interviews were conducted face-to-face guided by a list of questions and prompts developed by the researchers. Participants were encouraged to talk openly and to tell of their own experiences. The interviews ranged from 10 minutes to 45 minutes in length. The interviews were conducted by one of the three researchers. When each interview was completed, the researchers informed the participant about contacting them a second time to follow-up about the data to ensure that the researchers' interpretations are accurate with what the participants wanted to share with the researchers.

These are the steps of Colaizzi's process for phenomenological data analysis (Sanders, 2003; Speziale \& Carpenter, 2007).

- Each transcript was read and re-read to obtain a general sense about the whole content.

- For each transcript, significant statements that pertain to the phenomenon under study was extracted. These statements were recorded on a separate sheet noting their pages and lines numbers.

- Meanings were formulated from these significant statements.

- The formulated meanings were sorted into categories, clusters of themes, and themes.

- The findings of the study were integrated into an exhaustive description of the phenomenon under study.

- The fundamental structure of the phenomenon was described.

- Finally, validation of the findings was sought from the research participants to compare the researcher's descriptive results with their experiences.

The study was conducted on current and recent college students, including eight participants who follow a vegetarian diet and eight participants who include meat in their diet. Students were recruited through word of mouth. A private and quiet location on campus or near campus suitable for the participants was used for interviews. The researchers listened to the recordings of the interviews and transcribed them into a Word document verbatim to identify themes. After the data was transcribed, the recordings were deleted. Demographics were compiled into a table and analyzed using descriptive statistics. Themes were determined, compiled, and described based on frequency repeated.

\subsection{Reliability and Validity}

The participants answered demographic items and open-ended questions. The interview guideline was developed by the researchers and based on a review of the literature. The tool was reviewed by two peers and two nursing faculty to obtain face-validity.

\subsection{Informed Consent and Confidentiality}

Each participant was given a consent form to sign prior to the interviews. The responses to the questions were recorded without the name of the participant, and the consent forms were placed in a separate folder from the transcribed interviews. Approval from the Institutional Review Board (IRB) was received.

All collected data for this research was submitted to the School of Nursing to be stored electronically for three years. The School of Nursing staff scanned the data into the computer and stored it on discs in a locked cabinet in a locked storage room. Only the nursing administrators or the research coordinators will have access to the stored records.

\section{Results}

From this study, there were nine common themes that emerged among the participant interviews. From these themes, five were from the vegetarian and vegan interviews, and four were from the meat-eating interviews. The vegetarian and vegan themes were limitation of the diet (1), health effects (2), confusion and stigma about diet (3), reasons for choosing this diet (4), and more conscious of what they eat (5). The meat-eating themes were choices (1), health effects (2), mocking and ridiculing (3), and workout (4). Participants were labeled from P1 to P16.

\subsection{Demographics Information}

Table 1

\begin{tabular}{lll}
\hline Variable & Frequency (f) & Percentage (\%) \\
\hline Gender & & \\
\hline Male & 6 & $37.5 \%$ \\
\hline
\end{tabular}




\begin{tabular}{cll}
\hline Female & 10 & $62.5 \%$ \\
\hline Age Group & & \\
\hline $18-20$ & 6 & $37.5 \%$ \\
\hline $21-23$ & 9 & $56.25 \%$ \\
\hline $24-27$ & 1 & $6.25 \%$ \\
\hline Ethnicity & \\
\hline White/Caucasian & 12 & $75.0 \%$ \\
\hline Black/African American & 3 & $18.75 \%$ \\
\hline Hispanic/Caucasian & 1 & $6.25 \%$ \\
\hline Type of Diet & & \\
\hline Vegan/Vegetarian & 8 & $50.0 \%$ \\
\hline Meat-eating & 8 & $50.0 \%$ \\
\hline Length of Current Diet & & \\
\hline Less than 1 year & 2 & $12.5 \%$ \\
\hline $1-4$ years & 10 & $62.5 \%$ \\
\hline Lifelong & 4 & \\
\hline
\end{tabular}

Note. $(\mathrm{N}=16)$.

Overall there were more females than males in this study with the total percentage of women being $62.5 \%$ compared to $37.5 \%$ males (see Table 1). Majority of the participants were within the age ranges between $21-23$, with a $56.25 \%$ total. Most of the participants within this study identified as white or Caucasian descent with a percentage of $75 \%$. Half of the people surveyed claim to follow the vegan/vegetarian diet, while the other half eat meat. Many of the participants had been following their current diets for 1-4 years, with a grand total of $62.5 \%$.

\subsection{Analysis of Themes}

4.2.1 Themes for Factors Determining the Choice of Vegetarian Diet

\section{Limitations of Vegetarian/Vegan Diet}

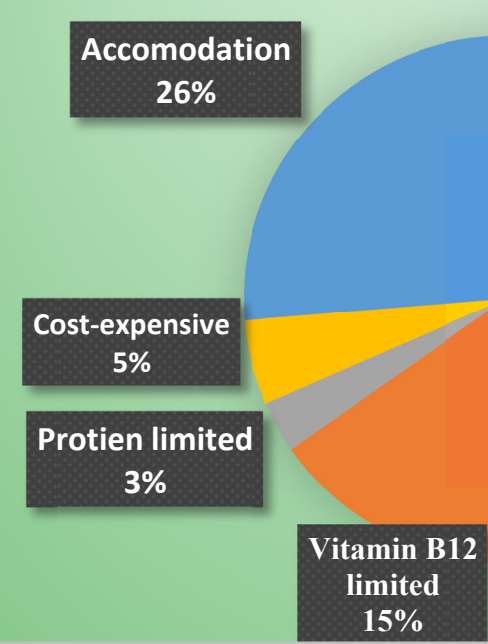

Choices

Limited

$51 \%$

Figure 1. Subtheme of limitations of vegetarian/vegan diet 


\subsubsection{Theme 1 for Vegetarian/Vegan Diet: Limitations of Vegetarian/Vegan Diet}

Limitations was the major theme that was noticed in the interviews for vegetarians and vegans. The main limitation discussed in the interviews was the choices (see Figure 1). Choices were also a noticeable theme in the meat-eating interviews that were conducted. Most participants mentioned several times in their interviews that there are not many food options for those following a plant-based diet. Participant one mentioned that, "Sometimes choices at restaurants or things like that are really limited. There are only like one or two choices maybe on the menu depending on where you go. So that could be difficult sometimes with friends if I go out to a restaurant, and there aren't really any choices or things like that."

There were a couple participants that mentioned that most of the time they bring their own food whenever they go somewhere. They said they do not want to burden people with having to make food they are able to eat. A few participants said that even when they are going out with friends, it is difficult because not all restaurants have options for vegetarians and vegans.

Another subtheme that was mentioned a lot was accommodation. Several people discussed how they have to bring their own food to social events, choose a specific restaurant they know has vegetarian or vegan options, or just eat after the event. Participants mentioned that it is sometimes difficult to make plans with friends because sometimes their friends want to go to a restaurant, but it doesn't have options for them. Then, they end up having to choose a different restaurant. Participant 2 talked about ways that he accommodates when he goes to restaurants or over to people's houses. This participant stated, "I usually let people know if they're inviting me over like hey, I'm vegan. I can bring my own food if you want me to. Thank you for inviting me over. I'm very okay with just sort of eating at home or eating later of something like that if there's not food there for me."

Some other subthemes that were mentioned in the interviews were the lack of vitamin B12, limited protein, and the high cost of vegetarian and vegan food. A lot of the participants talked about how they take vitamin B12 supplements because there are not really non-meat sources of this vitamin. It was also mentioned how there are not as many sources of non-meat protein. Finally, a lot of vegetables, fruits, and other vegetarian and vegan foods are more expensive. There are not a lot of cheap options for these foods.

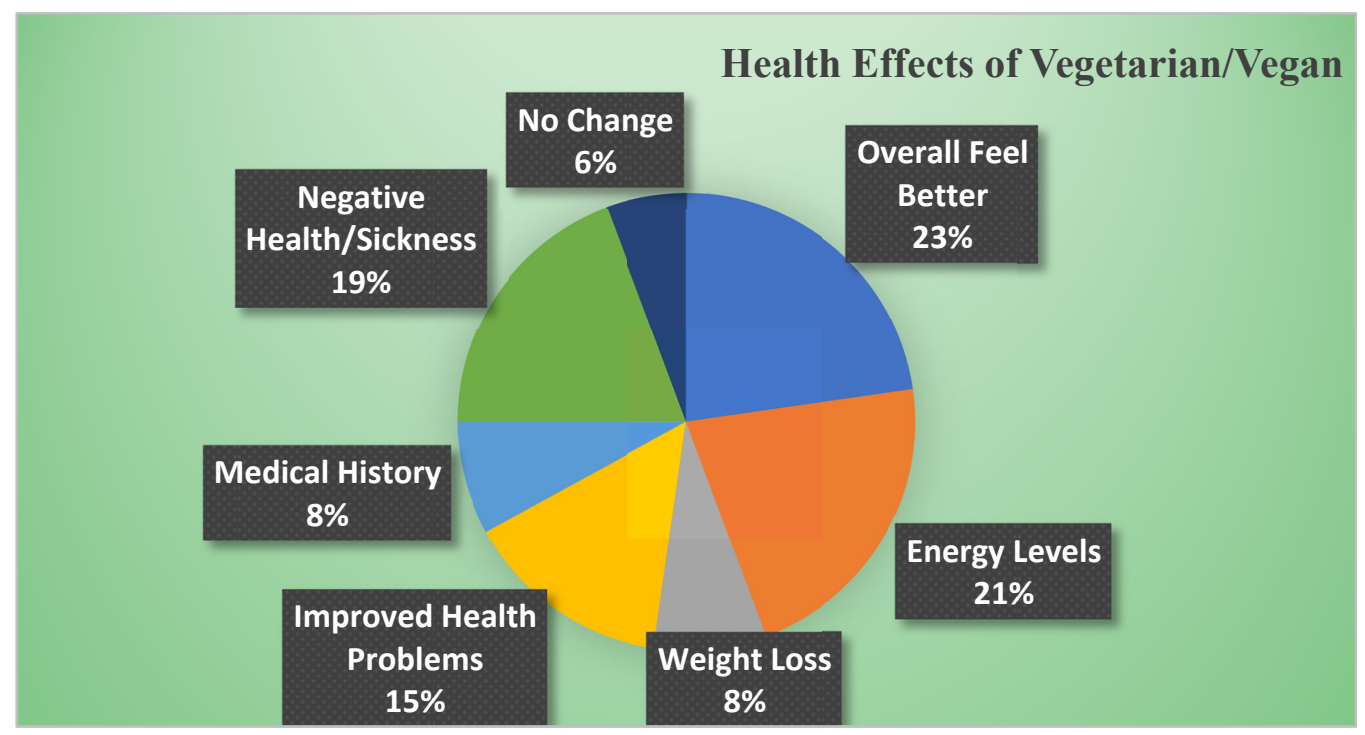

Figure 2. Subtheme of health effects of vegetarian/vegan diet

\subsubsection{Theme 2 for Vegetarian/Vegan Diet: Health Effects}

One aspect that was talked about a lot when interviewing vegetarians and vegans was health effects. A lot of people mentioned in their interviews that since following a vegetarian or vegan diet that the feel better in general (see Figure 2). Many participants talked about that this diet has given them a lot more energy. Participant 3 stated, "I feel a little bit more energized. I don't feel like as tired as I used to. . . Overall, just more upbeat and in a better mood." 
Some people talked about in their interviews how they do not get sick as often anymore. Participants talked about how they do not catch sicknesses as often. They also mentioned that when they are ill, it does not last as long as it used to. Participant 4 said, "something that I noticed is that like typically in the winter I'll probably get sick like two maybe three times. Last year, I only got sick once. I did notice that my immune system was better."

Some of the participants discussed how following this diet has helped to improve some of the medical problems they had. Participant 3 talked about how following a vegan diet has helped to reduce her ocular hypertension. Some other subthemes that were noticed in many of the interviews was the weight loss that people experienced, but there were a few that did not notice any changes in their health with following a vegetarian or vegan diet.

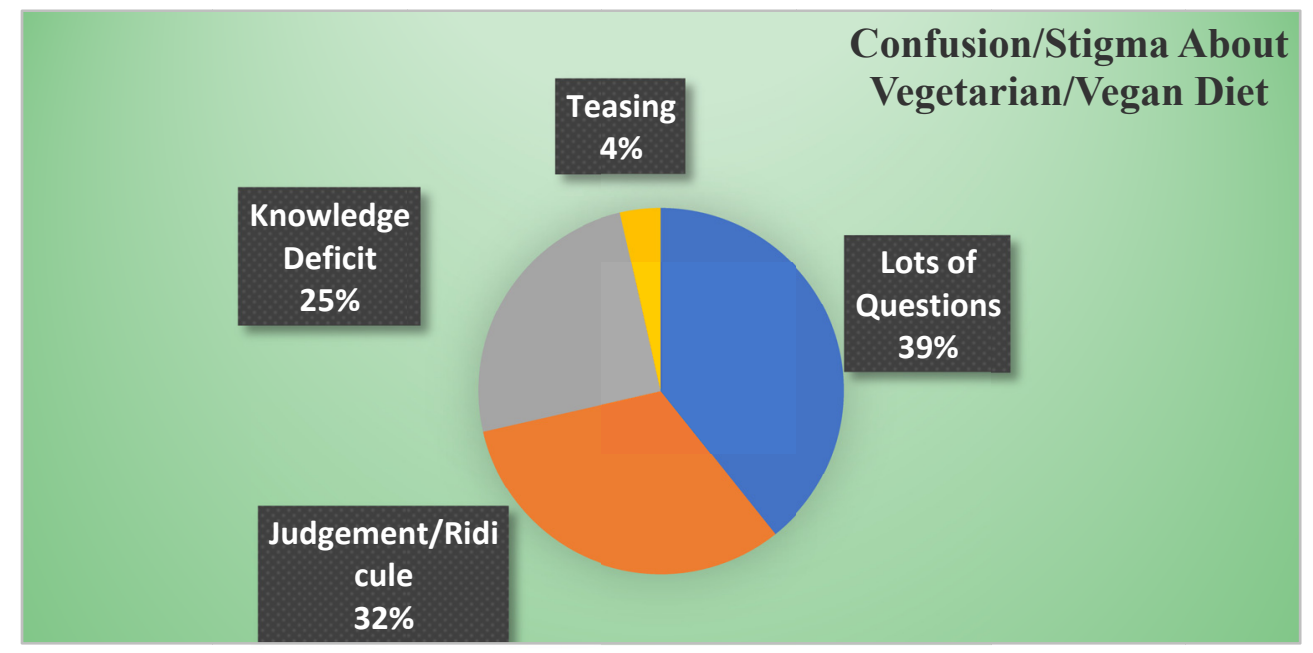

Figure 3. Subtheme of confusion/stigma about vegetarian/vegan diet

\subsubsection{Theme 3 for Vegetarian/Vegan Diet: Confusion and Stigma About Diet}

Both the vegan and vegetarian and the meat-eating interviews had a similar theme for their third most common themes regarding other people's opinions about their diet. For vegan and vegetarians, it could be summarized as confusion and stigma about their diet. As subcategories for this theme, the following were recognized: lots of questions, judgement/ridicule, knowledge deficit, and teasing (see Figure 3).

In most every interview, participants talked about the amount of questions that they received about their diet. Participant 4 stated, "I would say its just a lot of questions, which I don't have a problem with." Participant 5 said, "Sometimes, I get the most random questions by my family members." Many participants just talked about how many questions that they get about their diet.

Some of these questions were given in a judgmental way or were ridiculing. Participant 2 was asked, "Are you eating enough? Are you okay?" Participant 5 has been asked, "Ok, so do you want to eat grass?" Participant 5 was also told, "You're going to be malnourished." Other participants experienced disapproval from the people around them. Participant 1 was discouraged from their diet.

However, other questions or reactions just came from a lack of knowledge. Participant 4 was asked, "where do you get your protein? ...What do you eat? Do you just eat vegetables all day?" Participant 8 talked about how people would give her weird stares when they asked for just vegetables on their sub sandwich. Participant 7, who followed a vegetarian diet, talked about how people would be confused when they went to get coffee creamer and would ask if Participant 7 could actually have that. Participant 7 also said, "They're just genuinely confused by what it is."

Lastly, it was noted a few times that participants talked about teasing. Participant 7 said, "Either they're just straight up messing around and I know it and it's their personality." Participant 3 said, "My family still pokes a little fun about it." While this was not a main sub-theme that emerged, it was still stated by a couple participants. 


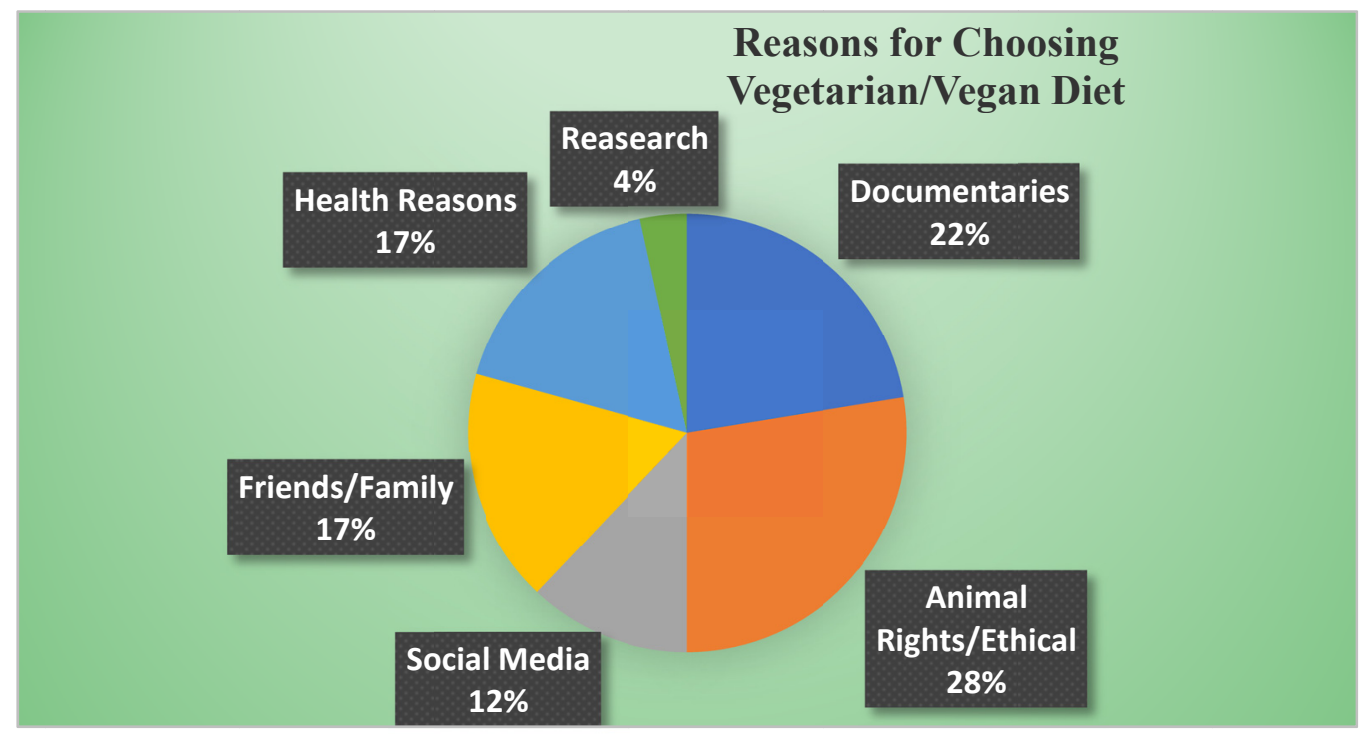

Figure 4. Subtheme of reasons for choosing vegetarian/vegan diet

\subsubsection{Theme 4 for Vegetarian/Vegan Diet: Reasons for Choosing Vegetarian/Vegan}

Another theme that was noticed when comparing our interviews for those with vegetarian or vegan diets was their reasons for choosing their diets. There was a variety of reasons why the participants decided to follow these diets. One of the top reasons had to do with animal rights and ethical reasons. Participant 7 stated, "With me working with Animal Control, I just think it's like the word cognitive dissonance. You save some, you eat others and it's just like it doesn't really make sense if you think about it. So, I just took that moral dilemma right off my plate."

A lot of the people that were interviewed mentioned that one of the major influences for them was some of the documentaries that they watched. A couple participants mentioned that one of the documentaries that they watched was on Netflix called "What the Health." Other reasons why people chose to follow these diets was social media, friends, family, health reasons, and research they found (see Figure 4).

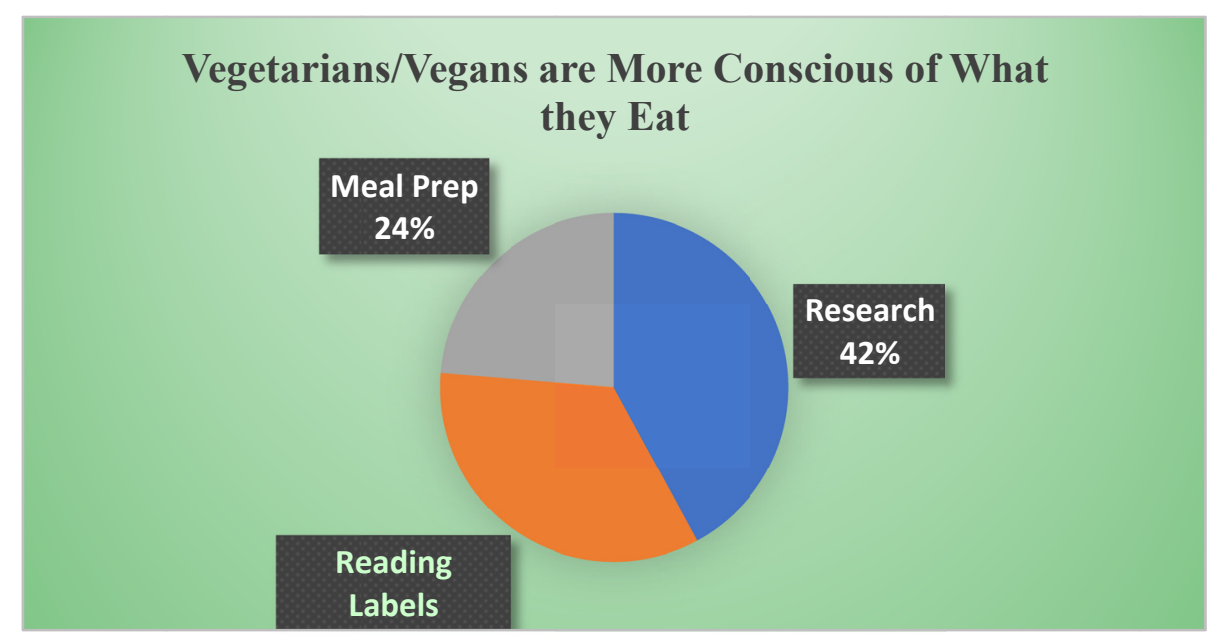

Figure 5. Subtheme of more conscious of what they eat for vegetarian/vegan diet

\subsubsection{Theme 5 for Vegetarian/Vegan Diet: More Conscious of What They Eat}

Another trending theme throughout the vegan/vegetarian interviews was how the people following these diets must be conscious of what they eat (see Figure 5). As in they now have to know what's inside the foods, they are 
going to consume to verify it is appropriate for their diet; whether that was in just eating foods that were 'vegan/vegetarian' or within the macro requirements for their nutritional needs. Participant 1 for example needed to research things in their food for both health and to make sure it fit; it "made me more aware of what's in my food, what I'm eating, and helped me become kind of motivated to look into nutrition and what I need in my diet." Participant 7 also added that, "when you start out it makes you more aware... I started reading labels a lot more than I ever had." In general, following this diet means being very aware what's in your food, but Participant 5 stated that "you can find things you'd never expect to be vegan or things that aren't. I'm still learning."

Participant 8 mentioned their natural curiosity of the food they ate. They mentioned how they "really conscious about what I eat all the time," and how "it's made me think a lot about what everything has in it and how it's made." This stemmed their research and ultimately led to their decision to transition into the vegetarian diet.

Whereas some of the participants noted the difficulties to find food appropriate to their diets and stated they need to bring their own food many times. Participant 1 even stated, "I have to prepare if I want to eat for myself." Participant 4 sympathized as they too "bring my own food to things... I take food everywhere that I go."

The research is also related to understanding the concepts of what a vegan/vegetarian can consume. Participant 5 shared their struggle when they mentioned how "it kind of takes a while to understand what I can and can't have," in regard to their friends; they admitted that "the thing that upsets me the most, is that the people don't do their own research."

\subsubsection{Themes for Factors Determining the Choice of Meat Eaters}

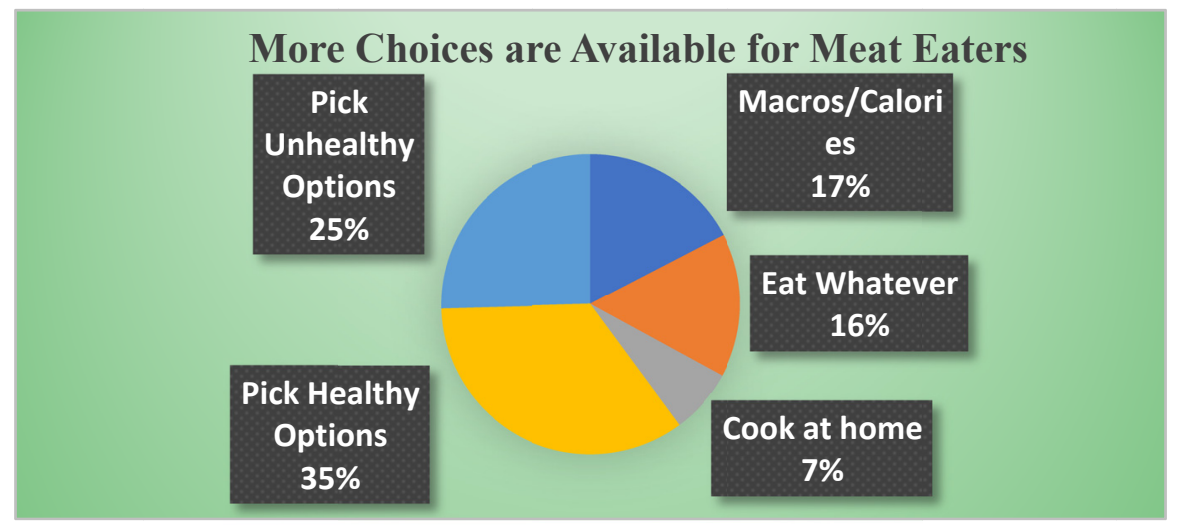

Figure 6. Subtheme of choices for meat-eating diet

\subsubsection{Theme 1 for Meat-Eating Diet: Choices}

Another theme found throughout the interviews with participants following the meat-eating diet, was regarding choices with their food options. Most of the participants talked about whether their regular food choices were healthy or not; with $35 \%$ of them believing they were healthy and $25 \%$ believed they were unhealthy (see Figure 6). The margin of these two percentages aren't very far apart, but the interviewees who claimed to eat unhealthily, were aware and desired to change their habits. For example, Participant 9 was constantly saying things like, "I wish I was more of a healthy eater," or "I just really wish I ate healthier."

This occurrence is not due to the lack of healthy choices, but the ability to eat whatever they want too $(9,12,14$, 16). Or in the words of participant 12, "I give into caving and temptations," or Participant 14 when they said, "I usually decide what I eat just like if it looks good." The trend out of these bad habits seem to be the choice to begin meal-prepping. The participants who actively meal-prep $(11,13)$ seem to have a conscious grasp of what macros and nutrients they need to live an active lifestyle. Participant 13 said it best when they stated: "I used to track calories, but I've found that it is better for you to track your macros. So, that's what I do now. It's because the three main sources of food for your body and so in order, to feel good not necessary to lose weight or gain muscle, to be getting the correct amount of energy my body needs. I have to meet all of those three macros, which are proteins, fats and carbs." 


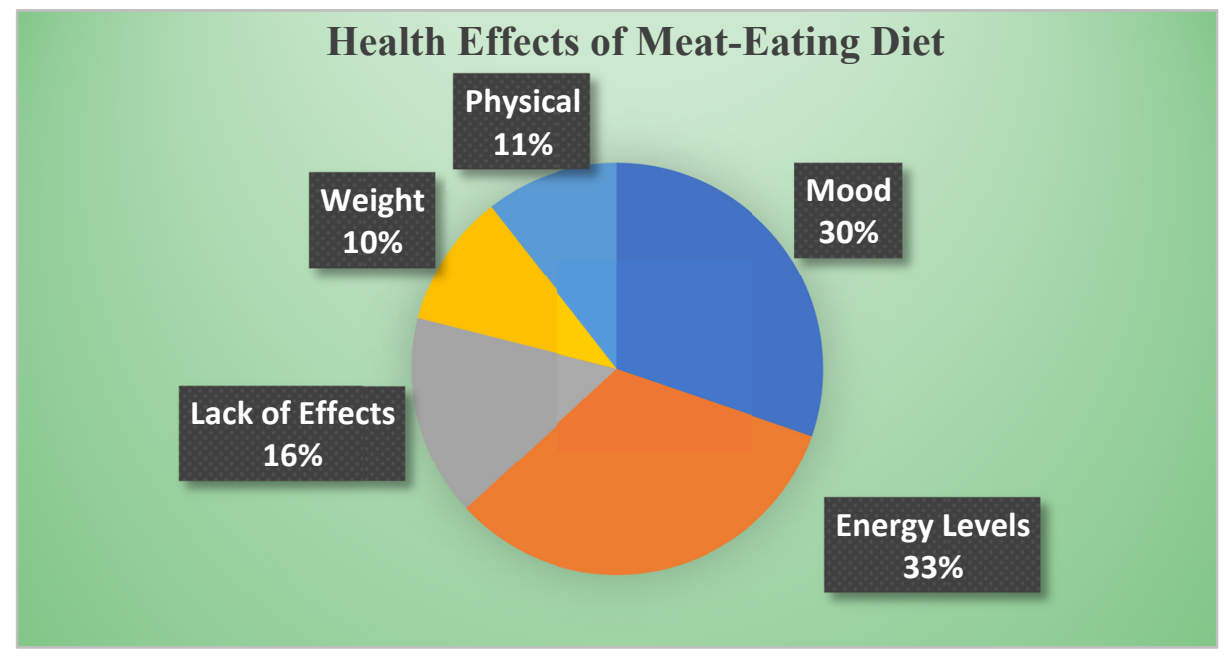

Figure 7. Subtheme of health effects of meat-eating diet

\subsubsection{Theme 2 for Meat-Eating Diet: Health Effects}

Another theme discovered through the interviews conducted was on the health effects noticed of people following the meat-eating diet. The majority of the participants discussed findings related to their energy levels, $33 \%$, and their mood, 30\% (see Figure 7). Participants rationalized their energy levels primarily with what they consumed. For example, Participant 9 stated, "Just really unhealthy foods that make me feel honestly very tired, and it's probably not the best." Additionally, Participant 11 claimed that they would "still feel sluggish" and that they noticed they were "always tired;" but with a change in diet that they found that it "has been helpful for me; with my energy and my focus..." A change of diet also helped Participant 13 as before their change in diet, they "weren't getting enough energy;" but now, they claimed that their "workouts are so much easier for me now. I feel so much better."

Almost all the participants discussed how their diet affected them emotionally, with similar results as the energy levels, as in healthy diets seemed to be more upbeat. Participant 11 summarized this concept when they stated, "when you're eating healthy you just feel vibrant, upbeat, like you have a pep-to-your-step, and your just healthier I think."

Still, there was a skeptic to the relationships between their health effects and their diet. Participant 14 claimed that they "haven't noticed it affecting my health," or "noticed any difference with it." Though this is the case, majority of the participants seemed to notice some sort of change with their health and their diet choices. Participant 15 shared most of the other interviewee's sentiments when they said, "You feel better when you eat the right things. Otherwise I feel tired and it's not good."

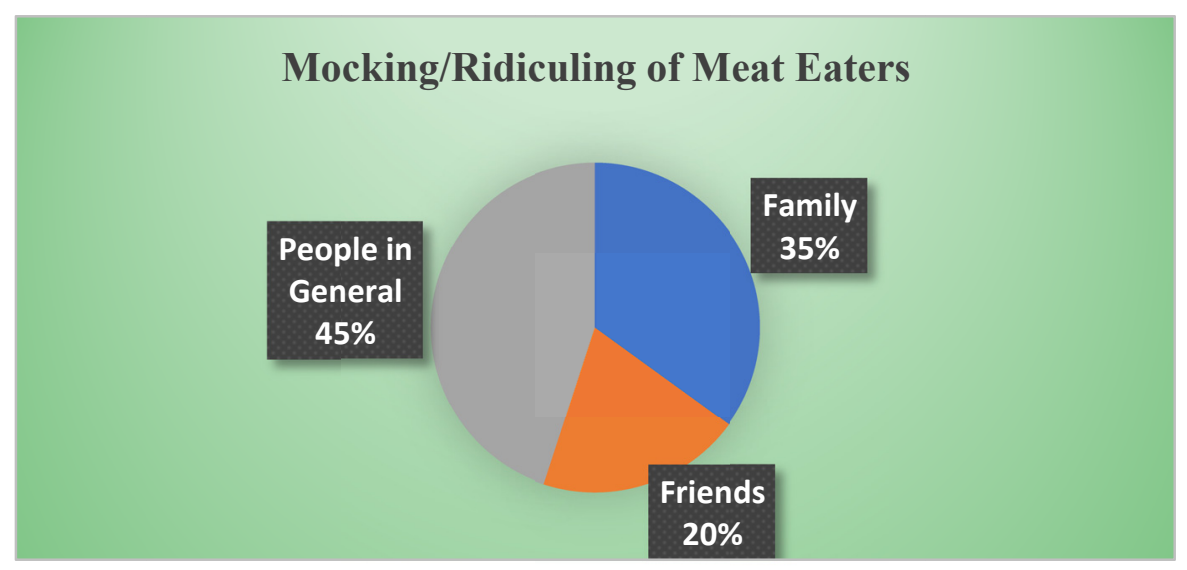

Figure 8. Subtheme of mocking/ridiculing of meat-eating diet 


\subsubsection{Theme 3 for Meat-Eating Diet: Mocking/Ridiculing}

The participants who followed a meat-eating diet also experienced mocking and ridiculing for their dietary preferences. They experienced this from friends, family and just people in general (see Figure 8). Participant 9 described a situation with some of their friends that are foreign exchange students, "They'll be like what are you doing? That's disgusting. You're unhealthy. That's junk food." Participant 10 talked about how their family finds the diet that they follow kind of gross. Participant 12 talked about how their family ridiculed people who did follow vegan or vegetarian diets and they stated, "which kind of swayed their interest."

Several participants expressed people calling their food unhealthy. Participant 14 also experienced people ridiculing his dietary preferences and calling them unhealthy. Participant 14 stated that, "A lot of people are like what's way more sugar than you are supposed to be, consuming in a day." Participant 15 also said that a lot of people do not understand calories and stated, "'Yeah, that's why I don't eat ten Oreos.' Or something like that so a lot of people don't understand." These were the shared experiences of feeling ridiculed or mocked for either the diet that they follow of the one that they would want to follow.

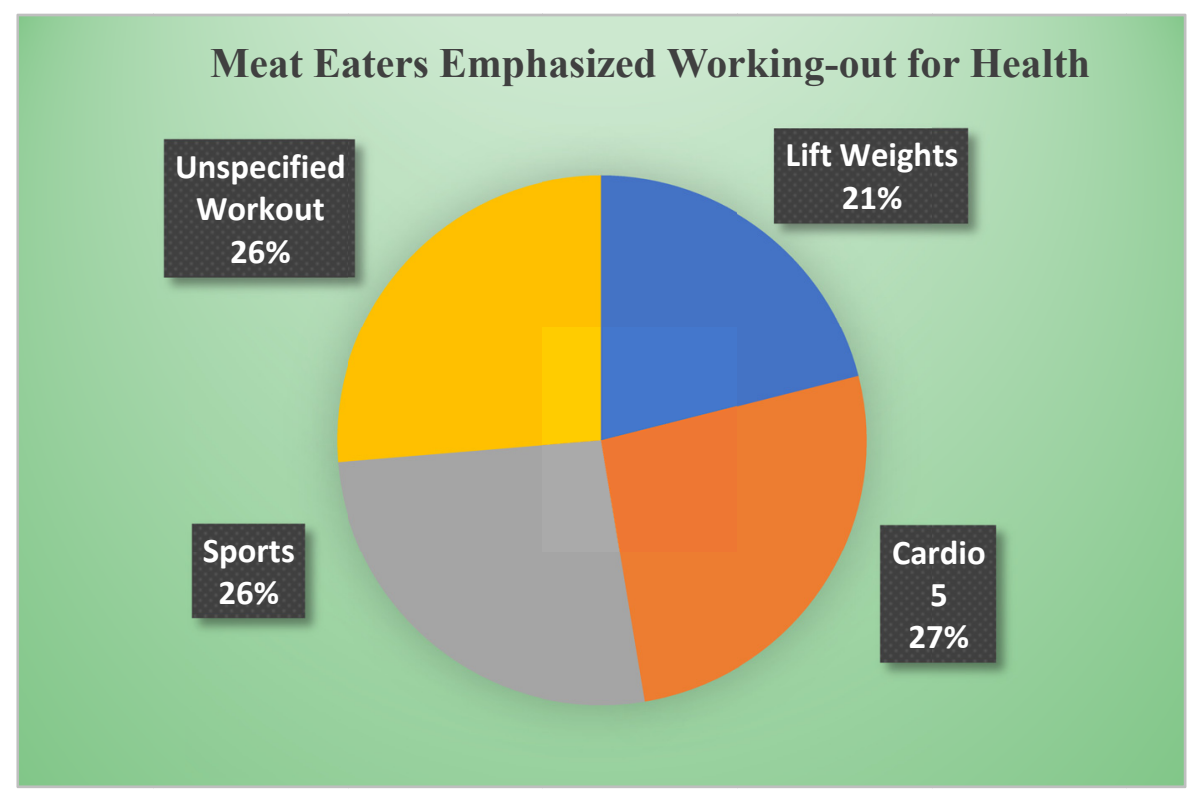

Figure 9. Subtheme of workout for meat-eating diet

\subsubsection{Theme 4 for Meat-Eating Diet: Workout}

The last theme that was recognized from the interviews was working out and exercising. Participants talked about past activity, current, and plans for the future. Sub-themes that emerged were lifting weights, cardio, sports, and unspecified workouts (see Figure 9). Participant 9 said, "I was also exercising daily." Participant 11 talked about how, "I was active, playing basketball, working out" and then about how they used to run cross-country. They discussed their current activities of playing basketball and how when its warmer they will start doing high-intensity interval training.

Participant 12 stated, "I work out 5 days a week" and later clarified that it was mostly weightlifting. Participant 13 also described their normal schedule as, "with softball I have morning conditioning, a hitting in the afternoon, practice in the evening." Participant 14 talked about staying active with volleyball and basketball and how they used to work out more and want to go back to that in the future. Participant 10 talked about, "exercising three or four times a week." Participant 11 talked about playing basketball and lifting. Many participants talked about various patterns of working-out and exercising. 


\subsubsection{Comparison of Data}

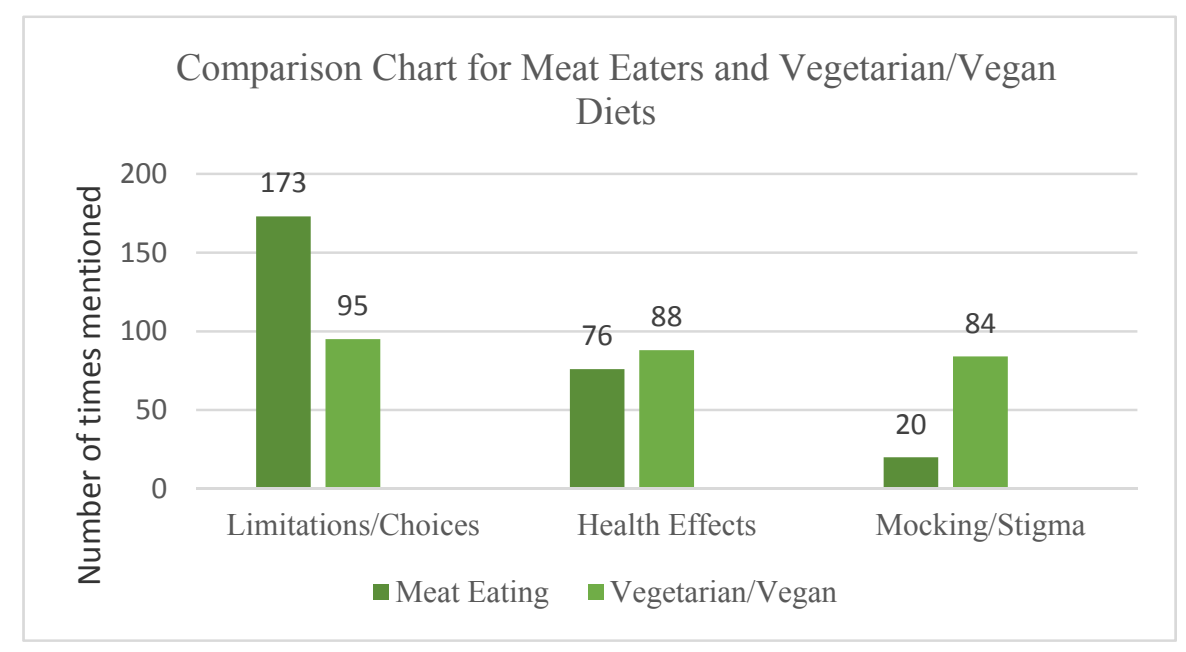

Note: $\mathrm{N}=16$ (Meat Eating $=8$, Vegetarian $/$ Vegan $=8)$

Through deduction of the final results of this study, it was determined that there are three themes that fit for comparison between the meat-eating and vegetarian and vegan diets. It was shown that meat-eating diets were prone to have a lot more choices as it was mentioned 173 times throughout all of the meat-eating interviews. In comparison, vegetarians and vegans were more likely to discuss how they were limited on food options, as it was mentioned 93 times throughout those interviews. Within these interviews it would appear that meat-eating diets have more choices, and vegetarian and vegan diets are more limited on their food options. Another comparison was health effects from following a meat-eating diet or following a vegetarian and vegan diet. In the meat-eating interviews, it was mentioned 76 times whereas in the vegetarian and vegan interviews it was mentioned 88 times. Within these interviews, it would appear that vegetarians and vegans have slightly more health effects than meat-eating diets. Lastly, the mocking and ridicule experienced from those following the meat-eating diets can be compared to the confusion and stigma experienced by those following the vegetarian and vegan diets. Within the meat-eating diets, they discussed mocking and ridicule about their diets 20 times. While in the vegetarian and vegan diets, they claimed confusion and stigma about their diets 84 times. Through this comparison it would conclude that vegetarians and vegans have more issues with confusion and stigma about their diets, than meat-eating diets have in regard to being ridiculed and mocked.

\section{Limitations}

Limitations included a small sample size, homogeneity of sample, and inexperienced researchers. As this study is designed to only have 16 participants, the results were limited by the sample size. The sample was very homogenous since all participants are currently or had attended this college. Another limitation was trying to find vegetarians or vegans who followed the diets strictly.

\section{Clinical Implications}

This study helps to inform the healthcare community about the factors that lead a person to follow a vegetarian diet versus a meat-eating diet. This can help to increase the understanding between healthcare providers and their patients. Understanding why patients pick certain diets helps healthcare providers know how to provide thorough dietary education to patients.

\section{Recommendations}

The need for further studies on this topic is evident from the literature review. In this study, even though people eating vegetarian diets claim to feel better, have increased energy, and less health problems, further studies are needed with a larger sample size and in multiple locations. This can help to understand people's experiences with their diets, whether meat-eating or vegetarian or vegan.

\section{Conclusion}

Published literature focused primarily on examining the association between diets and health outcomes. In this 
study, the factors determining the choice of vegetarian vs. meat-eaters include variations in choice, difference in perceived energy levels, perceived health concerns, and ethical issues. Vegetarian diets have become a popular dietary alternative; however, stigma related to its use was eminent in this study. Most public eating places do not have a good choice of vegetarian meal. People have to buy their vegetarian grocery and cook it themselves, which keep them away from some social situations. Vegetarians are thought of as maintaining their health by cautious eating and sedentary way of life whereas meat-eaters preferred to exercise or workout to be healthy. Compared to meat-eaters, vegetarians read labels and are more cautious of what they eat. Overall, meat-eaters had more choices in their diet whereas vegetarians and vegans struggled with limitations.

\section{References}

Brown, D. D. (2018). Nutritional considerations for the vegetarian and vegan dancer. Journal of Dance Medicine \& Science, 22(1), 44-53. https://doi.org/10.12678/1089-313X.22.1.44

Chiu, Y.-F., Hsu, C.-C., Chiu, T. H. T., Lee, C.-Y., Liu, T.-T., Tsao, C. K., ... Hsiung, C. A. (2015). Cross-sectional and longitudinal comparisons of metabolic profiles between vegetarian and non-vegetarian subjects: A matched cohort study. British Journal of Nutrition, 114(8), 1313-1320. https://doi.org/10.1017/S0007114515002937

Craddock, J. C., Probst, Y., \& Peoples, J. (2016). Vegetarian nutrition - comparing physical performance of omnivorous and vegetarian athletes. Journal of Nutrition \& Intermediary Metabolism, 4(C), 19-19. https://doi.org/10.1016/j.jnim.2015.12.219

Cramer, H., Kessler, C. S., Sundberg, T., Leach, M. J., Schumann, D., Adams, J., \& Lauche, R. (2017). Characteristics of Americans choosing vegetarian and vegan diets for health reasons. Journal of Nutrition Education \& Behavior, 49(7), 561-567. https://doi.org/10.1016/j.jneb.2017.04.011

Dinu, M., Abbate, R., Gensini, G., Casini, A., \& Sofi, F. (2017). Vegetarian, vegan diets and multiple health outcomes: A systematic review with meta-analysis of observational studies. Critical Reviews in Food Science and Nutrition, 57(17), 3640-3649. https://doi.org/10.1080/10408398.2016.1138447

Godos, J., Bella F., Sciacca, S., Galvano, F., \& Grosso, G. (2017). Vegetarianism and breast, colorectal and prostate cancer risk: An overview and meta-analysis of cohort studies. Journal of Human Nutrition and Dietetics, 30, 349-359. https://doi.org/10.1111/jhn.12426

Pender, N., Murdaugh, C., \& Parsons, M. (2006). Health promotion in nursing practice (5th ed.). Upper Saddle River, NJ: Pearson Prentice Hall

Piccoli, G. B., Clari, R., Vigotti, F. N., Leone, F., Attini, R., Cabiddu, G., ... Avagnina, P. (2015). Vegan-vegetarian diets in pregnancy: Danger or panacea? A systematic narrative review. An International Journal of Obstetrics \& Gynecology, 122(5), 623-633. https://doi.org/10.1111/1471-0528.13280

Sanders, C. (2003). Application of Colaizzi's method: Interpretation of an auditable decision trail by a novice researcher. Contemporary Nurse Journal, 14(3), 292-302. https://doi.org/10.5172/conu.14.3.292

Speziale, H. J., \& Carpenter, D. R. (2007). Qualitative research in nursing: Advancing the humanistic imperative (4th ed.). Philadelphia: Lippincott, Williams \& Wilkins

Trepanowski, J., \& Varady, K. (2015). Veganism is a viable alternative to conventional diet therapy for improving blood lipids and glycemic control. Critical Reviews in Food Science and Nutrition, 55(14), 2004-2013. https://doi.org/10.1080/10408398.2012.736093

\section{Copyrights}

Copyright for this article is retained by the author(s), with first publication rights granted to the journal.

This is an open-access article distributed under the terms and conditions of the Creative Commons Attribution license (http://creativecommons.org/licenses/by/4.0/). 